\title{
FTIR surface analysis for conservation
}

\author{
David Thickett ${ }^{1^{*}}$ (D) and Boris Pretzel ${ }^{2}$
}

\begin{abstract}
Several FTIR techniques are surface-sensitive. This sensitivity can be utilized to help with a wide range conservation issues. Examples from object degradation studies, conservation materials performance assessment and monitoring are presented. These are used to discuss the issues, advantages and information that can be drawn from studies. Glass degradation was quantified on the surfaces of cover glasses from important Nineteenth century daguerreotypes using Germanium attenuated total reflectance. The very limited information depth endowed by the high refractive index crystal gave good sensitivity when other techniques such as sectioning and scanning electron microscopy had failed to detect a gel layer present. The inner glass surface was always more deteriorated and the degree of deterioration inversely correlated with the distance between the inner glass surface and the daguerreotype or brass matt surface. Reflection-absorption FTIR microscopy has been combined with an excimer laser to determine the water content of a series of sections through the depth of a wax. A common wax used to protect metals has been found to take up liquid water in humid environments and hold that water close to or at the metal surface. An adjustable height gold mirror was used to bounce the IR beam many times across a surface, which can give extreme sensitivity. Haze and smoke generators are increasingly being introduced into displays or for special events or filming. Very low detection limits (1 ng) were found using gold coated glass slides to detect haze deposits.
\end{abstract}

Keywords: FTIR, ATR, Transflection, Wax, Haze, Glass

\section{Introduction}

Analysis can be transformational in conservation practice. There are three main areas of preventive conservation where analysis is critical: degradation studies, conservation material performance studies and monitoring. Many phenomena occur at surfaces and surfacesensitive analyses are required. This paper presents an infra-red spectroscopy research example of each area, and explains why the very to extremely high surface sensitivity was of particular benefit to those studies. Access to analysis has widened over the past two decades. Instrumentation has developed and is generally less expensive, often easier to use and, in many instances portable and non-invasive. Surface analytical techniques have been used for heritage degradation studies for at least 20 years. Dynamic Secondary Ion Mass Spectroscopy has been used to follow the early stages of glass

\footnotetext{
*Correspondence: david.thickett@english-heritage.org.uk

${ }^{1}$ English Heritage, Rangers House, London SE108QX, UK

Full list of author information is available at the end of the article
}

deterioration [1]. Both $\mathrm{x}$-ray photoelectron spectroscopy and static and dynamic SIMS were used in the investigation of silver tarnish and lacquer impact on tarnishing $[2,3]$. The surface-sensitive Attenuated Total Reflection Fourier Transform infra-red spectroscopy, ATR-FTIR is widely used in conservation. It has been used to characterise almost every material used in cultural heritage and its conservation [4-6] with many examples in the presentations of the thirteen Infra-red and Raman User Group conferences. The surface sensitivity of ATR-FTIR has more recently been used to follow the chemical degradation of rubber and amber $[7,8]$. This technique is inherently surface sensitive, which can be utilised beneficially, but can also be an issue if bulk analyses are required [9].

\section{Degradation of daguerreotype cover glasses}

Daguerreotypes are amongst the earliest photographic methods. The technique produces silver nanoparticles on a silvered copper plate. The image is very fragile, sensitive to pollutants, and readily damaged by abrasion; daguerreotypes were generally produced in a protective

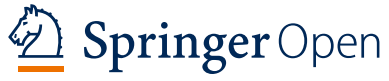

(c) The Author(s) 2020. This article is licensed under a Creative Commons Attribution 4.0 International License, which permits use, sharing. adaptation, distribution and reproduction in any medium or format, as long as you give appropriate credit to the original author(s) and the source, provide a link to the Creative Commons licence, and indicate if changes were made. The images or other third party material in this article are included in the article's Creative Commons licence, unless indicated otherwise in a credit line to the material. If material is not included in the article's Creative Commons licence and your intended use is not permitted by statutory regulation or exceeds the permitted use, you will need to obtain permission directly from the copyright holder. To view a copy of this licence, visit http://creativeco mmons.org/licenses/by/4.0/. The Creative Commons Public Domain Dedication waiver (http://creativecommons.org/publicdomain/ zero/1.0/) applies to the data made available in this article, unless otherwise stated in a credit line to the data. 
cover. The image was protected by cases composed of an outer wood, leather and velvet box, with a brass cut-out matt and glass cover over the actual image. English Heritage's Down House, the home of Charles Darwin, has eight daguerreotypes of Darwin's family that have exhibited degradation with the appearance of crystals on the glass on the inside of the cases which impede viewing the image. Rapid acceleration of the formation of crystals, elicited investigation into the degradation mechanism of the glass. The aim of this work was to provide insight into the degradation mechanism to allow changes to slow the degradation and allow continued display of the Daguerreotypes in their original context. The spacial distribution of the glass degradation was of particular interest. Before the cases were disassembled, the glass-to-daguerreotype and glass-to-matt depth was analysed at over 30 points for each using a laser displacement sensor (Acuity AR600). The effect of glass refraction was compensated by running a calibration afterwards at each point using the cover glass and feeler gauges of 20 to $508 \mu \mathrm{m}$ thickness to set the distance between the cover glass and an aluminium mirror. In some instances the cover glass had to be turned to account for geometry.

The salts on the glass surfaces were initially analysed by removing four samples from each plate with a chemically sharpened tungsten needle, placing them on a diamond ATR and analysing with a Perkin Elmer Spectrum 65 FTIR spectrometer. Later analyses of over 30 salts on each plate were taken with a IR microscope (Continuum on Thermo Is-10 FTIR) in total reflection mode. All salt crystals were determined to be sodium formate. Analysis of multiple samples is important in this instance. Each plate has hundreds of crystals present and several authors have reported analyses with mixed ions and other species present on glass surfaces [10-12]. Whilst representative crystals were selected for the initial analyses (considering colour, morphology and size), the same compound can give different size and morphology crystals depending on the environmental conditions. This FTIR microscope is not sensitive to chloride as the MCT detector used cuts off at $650 \mathrm{~cm}^{-1}$. In these instances SEM-EDX (FEI Inspect) analyses of three of the plates and ion chromatography (Dionex 600 AS14A column and carbonate/bicarbonate eluent), analyses of $18.2 \mathrm{M} \Omega \mathrm{cm}^{-1}$ water extracts of 7:3 IMS:water swabs detected very low concentrations of chloride present, less than $0.3 \%$ of the other anions. Additionally, when analysing directly on a glass surface, the characteristic $1100 \mathrm{~cm}^{-1}$ absorption bands of sodium sulfate overlap the glass silicate bands making it difficult to discern the two. Nethertheless, very low levels of sulfate were detected (less than $1 \%$ of total anions present). One of the glass plates was replaced in 1996, with a modern plate glass. Although this had visibly less crystals on the surface than the historical glass plates, there were still a great number present.

The modern replacement glass was cut into pieces and a series of cross sections produced, by embedding in epoxy and polishing. Two of the original glasses had small chips removed from a corner and were similarly processed. The cross sections were examined with scanning electron microscopy (FEI Inspect). Surprisingly, with the number of sodium formate salts present, no visible gel layers could be imaged. The inside, outside and cross sections' glass surfaces were analysed with a Germanium ATR head on the FTIR microscope. Germanium ATR crystals have the highest refractive index of widely available ATR crystals and hence, shallowest information depth. At $1100 \mathrm{~cm}^{-1}$, the information depth was calculated to be $0.5 \mu \mathrm{m}$ using the equation derived by Harrick [13] and a refractive index of 2.3 for glass and 4.0 for Germanium. The degree of glass degradation can be analysed with the splitting factor (difference in maxima wavenumbers) for the $\mathrm{Si}-\mathrm{O}-\mathrm{Si}$ and $\mathrm{Si}-\mathrm{O}-\mathrm{Na} / \mathrm{K}$ bands around 1100 and $970 \mathrm{~cm}^{-1}$ [14].

\section{Microcrystalline wax applied to indoor metals}

Renaissance Wax, a blend of Cosmoloid H80 and BASF wax A, is widely used in metals conservation [15]. Previous research [16] has shown that after a short protective period, it can actually increase the rate of corrosion for ferrous metals under high RH conditions. The wax was exposed on carbon steel pieces in the Secret Wartime Tunnels under the English Heritage site, Dover Castle. The previous research indicated that water was present below the surface of the wax. More precise localisation of the water was attempted to understand the poor performance observed for the wax coating. Additionally, experiments were run to understand at what $\mathrm{RH}$ water appears inside the wax and how this water evaporates when the RH falls. This information is critical to understanding in which environments the wax may perform well. Analysis of the wax coatings with a Nicolet Inspect IR microscope running off a Nicolet 360 Avatar FTIR was undertaken in two modes; reflection-absorption and with a silicon ATR head. The ATR spectra showed a weak water absorption band at $1645 \mathrm{~cm}^{-1}$, whilst that from the reflection-absorption (analysing the whole depth of the wax and reflecting from the steel surface) showed much stronger water absorption in all instances. No Reststrahlen or derivative bands were observed in the reflection-absorption spectra and hence, no corrections were made [9]. Calculations with the Harrick equation and using 1.45 as the refractive index (RI) of the wax [17] and a silicon (RI 3.42) ATR head, indicated the information depth at $1644 \mathrm{~cm}^{-1}$ ( $v_{2}$ bending vibration of water) was $1.60 \mu \mathrm{m}$. As the wax deforms under very little pressure, it 
is difficult to ensure the ATR analyses have not depressed the wax surface, limiting the accuracy of this method.

In order to further investigate the presence of water in or below the wax on metal surfaces, a calibration for wax depth on steel was generated. Wax samples were then ablated with a UV excimer laser (Lumionics Excimer-500) in small steps and analysed with an IR microscope (Nicolet Inspect) running off a FTIR (Nicolet 360 Avatar). Studies have shown that no energy penetrates beyond the ablation depth for UV lasers in organic materials [18]. Wax samples were compacted between copper metal foil pieces on a steel plate (excised with lines at $100 \mu \mathrm{m}$ intervals), allowed to dry, and were determined to have no further mass decrease on a 5 figure balance. Multiple sheets were used to form samples, initially 0.5 to $5.0 \mu \mathrm{m}$ deep at $0.5 \mu \mathrm{m}$ intervals. After drying, the depth at several points was determined with two methods. The depth of the single or multiple metal sheets was known and checked with a micrometer. The micrometer was bridged backwards across the two metal sheets and opened until the guide bar touched the wax surface. This was viewed at $45^{\circ}$ with a digital microscope. The surface was also scanned with a profilometer (Innowep $\mathrm{GmbH}$ TRACEiT), producing a 3D profile of the surface and the two metal sheets. The depth below the sheets was calculated at several points. The infra-red spectrum was determined at several points on each depth division. The $1450 \mathrm{~cm}^{-1}$ absorption was used to construct a depth calibration.

As the actual measurements are taken on a corroded steel surface, interference from corrosion product spectra was considered. Analytical points were selected with the visible microscope before switching to IR mode and obvious corrosion was avoided, but the absence of low levels could not be assured. Spectra collected previously of the full range of reported corrosion products for iron [19] were re-examined. Figure 1 shows the main bands (over 10\% of the strongest absorption) and the bands of Renaissance Wax. The choice of band strength is appropriate considering the selection of analyses from areas with no corrosion visible under magnification.

The $1450 \mathrm{~cm}^{-1}$ band was mainly clear of overlap with iron corrosion product bands, with exception of the $1417 \mathrm{~cm}^{-1}$ absorption band of iron carbonate. This corrosion product has only very rarely been reported in atmospheric corrosion [20] and x-ray diffraction of the steel surfaces did not detect its presence [16].

A series of wax samples on mild steel coupons were exposed to atmospheres with $\mathrm{RH}$ values of 75 to $95 \%$ in $5 \%$ intervals above glycerol solutions in well-sealed glass containers for 3 months. Six coupons were exposed to each atmosphere. Three of the exposed coupons were then kept at $40 \% \mathrm{RH}$ above Prosorb for 3 months. This is the minimum $\mathrm{RH}$ measured in many historic buildings, including the 1084 rooms measured at 104 English Heritage buildings. Each coupon was then sequentially UV-ablated and the ablation pit analysed with reflectionabsorption FTIR after each ablation. If the water absorption at $1645 \mathrm{~cm}^{-1}$ was greater than $10 \%$ of the height of the wax absorption at $1450 \mathrm{~cm}^{-1}$, significant amounts of liquid water were considered to be present.

\section{Haze}

Haze is increasingly being used in heritage spaces for filming, events and exhibitions, with exposures from a few hours to several months. There has been no

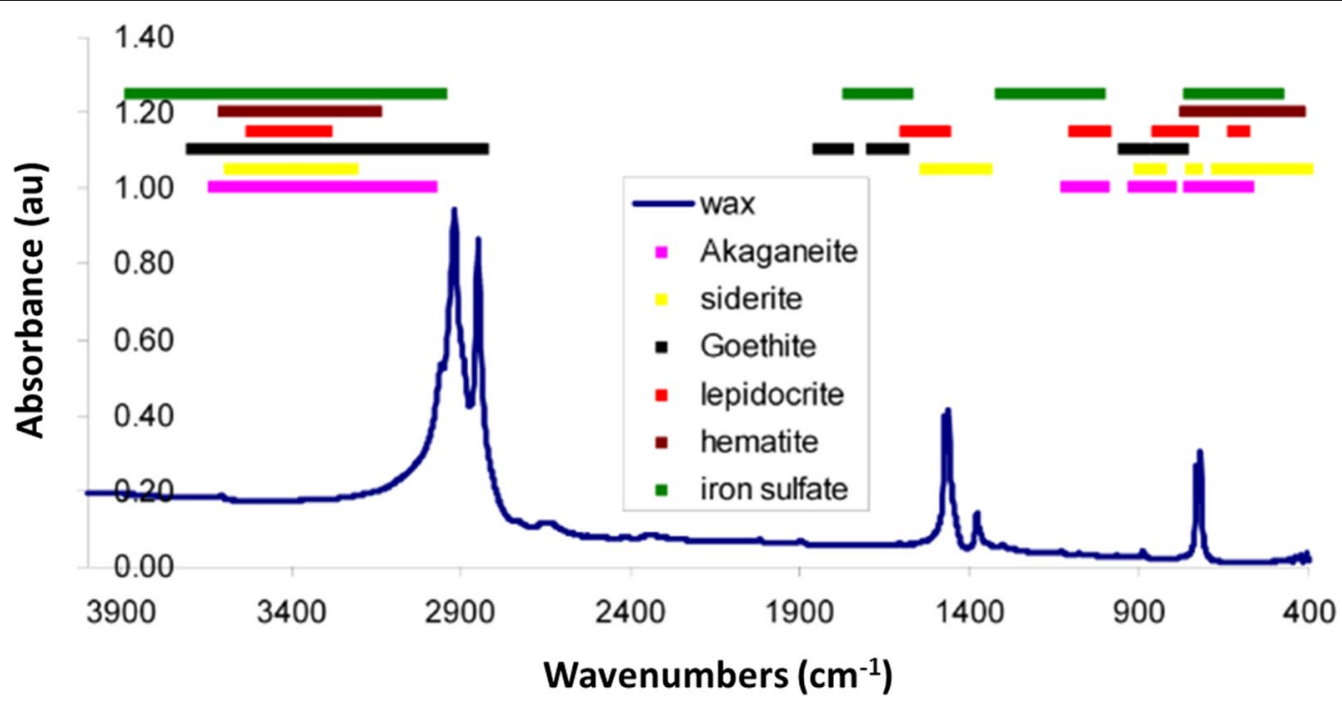

Fig. 1 Renaissance Wax spectra and iron corrosion product absorption bands widths (at 10\% maximum peak height) 
published research on the impact of the haze materials on cultural heritage materials and only one report in the grey literature [21]. Two main types exist, those based on glycerol and water and those based on mineral oils. The generator vaporises small particles of the material that refract light in the room to achieve certain lighting effects. Some unpublished work has been undertaken by the National Trust, recommending mineral oil over glycerol and suggesting machines generating finer particles be used in non-sensitive areas only [21]. One mineral oil generator was tested and protocols developed for its use to minimise the risk. A commercial analytical firm developed a detection method very rapidly. This method involved exposing two glass slides to the haze and taping them, faces together, for transport back to the laboratory. For analysis, the slides were extracted with solvent and analysed with FTIR. The method gave a detection limit of $1 \mu \mathrm{g}$ on the $37.5 \mathrm{~cm}^{2}$ combined surface of the slides. This was adequate for detection in the spaces the haze was used and adjacent spaces receiving high doses.

An exhibition at the Victoria and Albert Museum used lasers. The use of (Pea Soup) haze oil was requested to improve their visibility in a 4-month temporary exhibition space at high level. No Victoria and Albert accessioned objects were present in the space or immediately surrounding rooms, but there was concern about the deposition of haze in adjacent galleries. Several precautions were taken, including using the very minimum amount of oil, only when the exhibition was open. Other efforts to minimise the haze, involved sealing several adjacent spaces, using HEPA filtration and negative pressure to draw any haze back into the space through the exits and entrances. Air curtain was used on the exit. Similar monitoring was undertaken, but concerns still existed about the levels of haze deposition in surrounding collection galleries. The aim of this study was to determine whether the mitigation strategies had successfully prevented haze from being deposited on objects in the surrounding galleries. A method with improved detection limits was required. There were concerns about the low evaporation rate of the haze and potential long term deterioration effects.

A more sensitive method was developed using reflection-absorption (sometimes called transflection) IR spectroscopy. Gold-coated slides were exposed horizontally (parallel to floor) and vertically (perpendicular to the floor) and then analysed with reflection-absorption (Amplfi-IR accessory) on FTIR (Perkin Elmer 2000). The accessory uses a $5 \mathrm{~cm}$ diameter gold mirror, below the sample and the beam bounces multiple times from the mirror and the sample. The distance between the mirror and the sample determines the number of bounces and hence, detection limit. A $40 \mu \mathrm{m}$ distance between the mirror and sample slide was selected to give good sensitivity but allow for some smaller dust particles on the slide surfaces. The distance was measured using a laser displacement sensor (Acuity AR600). The thickness of the gold coating was assessed as adequate, as no glass absorption bands were observed in the background spectra. Such bands would complicate the analysis if present.

Microscopy of the exposed slides showed large particles up to $120 \mu \mathrm{m}$ on the horizontally exposed slides, and nothing above $30 \mu \mathrm{m}$ was observed on the vertically exposed slides. Tests with air dusters could not remove the larger particles. A method of attaching the slide upside down to the top of a vibratory mill and running for $2 \mathrm{~min}$ at $300 \mathrm{rpm}$ appeared effective. This removed particles down to $30 \mu \mathrm{m}$. Good quality spectra were produced for five of the nine slides, and one slide had a weak spectrum, but this only contained one of the three strong IR bands of the mineral oil.

The slides had been exposed for almost 60 days and large amounts of dust were visible. The IR spectra indicated the dust was mainly proteinaceous, likely to be either skin or woollen fibres. Haze is more commonly used for single events and the general exposure period would be much shorter, meaning much less deposited dust. Images were collected under $100 \times$ magnification and processed with Image-J for percentage coverage and a length ratio for each particle. This indicated that fibres (longest measurement, L/shortest measurement, $\mathrm{S}>3$ ) composed of approximately $1-4 \%$ surface area coverage, particles $(\mathrm{L} / \mathrm{S}<3)$ approximately $3-9 \%$. This indicated skin flakes were the major component of the dust. Two of the characteristic absorptions of the mineral oil (1370 and $712 \mathrm{~cm}^{-1}$ ) occurred in regions that had no peaks in the dust spectra (standard spectra for wool and human skin).

A calibration was produced by sequentially diluting the oil (Pea Soup) in petroleum ether $\left(80-100{ }^{\circ} \mathrm{C}\right)$. A controlled volume $(0.1 \mu \mathrm{ltr})$ was pipetted onto a $10 \mathrm{MHz}$ piezo electric quartz crystal (PQC) in the centre of the $5 \mathrm{~mm}$ diameter gold contact. The PQC was allowed to dry for 7 days in a fume cupboard and no spreading of the sample beyond the gold contact was confirmed with examination in UV light (the oil fluoresced strongly on both gold and quartz).

\section{Results and discussion \\ Daguerreotype cover glasses}

Typical ATR FTIR microscopy spectra are shown as Fig. 2.

In all instances, the inner glass surface was significantly more degraded than the outer surface. This indicates the environment inside the Daguerreotype packets is more corrosive than that in the showcase. As expected, the 


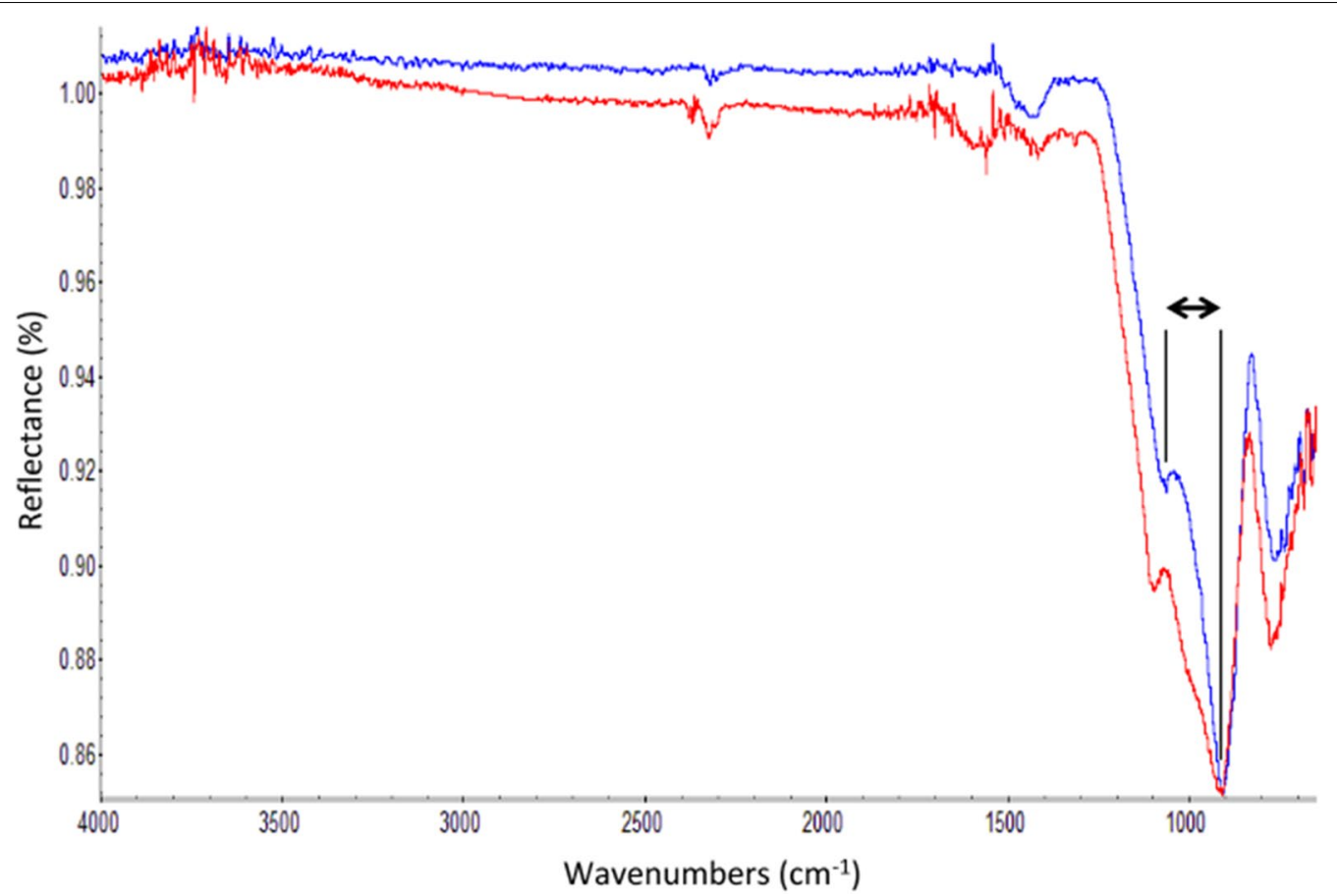

Fig. 2 ATR FTIR Spectra of degraded glass. The blue spectrum is the inner surface of the glass and shows a larger splitting factor than the red spectrum which is the outer surface

glass at the centre of the cross sections did not show distinct peaks and no splitting value could be determined.

Several spectra were taken at each point measured with the laser previously. The results for the interior glass splitting value versus glass to image or matt depth for two glasses (George and Annie) are shown in Fig. 3.

The degree of degradation of the glass, indicated by the splitting value, is greater for the shorter distance gaps above the matts for both glasses. There is slightly less degradation for the glass from Annie than that from George above the matt $(10-40 \mu \mathrm{m})$. The glass above the deeper image rebate $(230-430 \mu \mathrm{m})$ is less degraded and that from Annie is significantly less degraded than that from George. There is a rough negative correlation with depth and the slopes are different for the smaller glassto-matt and much deeper glass-to-image analyses. The slopes are different for each of the two glass plates shown. This may indicate differences in the environment inside the two packets or differences in glass susceptibility due to different glass compositions. Records as far back as the 1930s indicate both Daguerreotypes were stored or displayed together, meaning the external environment was the same. The glass on the exterior of the Daguerreotype packet from George was slightly more degraded $\left(61-70 \mathrm{~cm}^{-1}\right)$, than that from Annie $\left(54-66 \mathrm{~cm}^{-1}\right)$, perhaps indicating a more susceptible glass composition if the environments were identical. Although, the condition of the glass when the packet was made is unknown and that from George could have been stored for longer before use or in more aggressive conditions. Additionally, the ingress of pollutants such as formic acid and water vapour depends on the exact sealing of the packets and will probably differ between them. Research on Daguerreotype glass degradation is very limited. Whilst much progress has been made in understanding buried glass deterioration [22], there are few studies of the atmospheric processes occurring on historic glass compositions. These have been reviewed [23]. The deterioration within a Daguerreotype packet is clearly more rapid than in the ambient air around the packet and more complex. The single published study of Daguerreotype glass deterioration stated it is rare to see patches of weeping glass directly above the matt' and 'more common to see clusters of droplets in the more central portions' [24]. These results appear to be based on visual examination, although the authors characterised the corrosion products and bulk glass chemistries and mentioned undertaking SIMS and microRaman analyses, which were not reported or published subsequently. This result contradicts that work and emphasizes that although visual examination is a valuable first step, it can be misleading. This may have important ramifications for understanding the deterioration mechanism. For example, both accelerating pollutant ingress (such as formic acid) and 


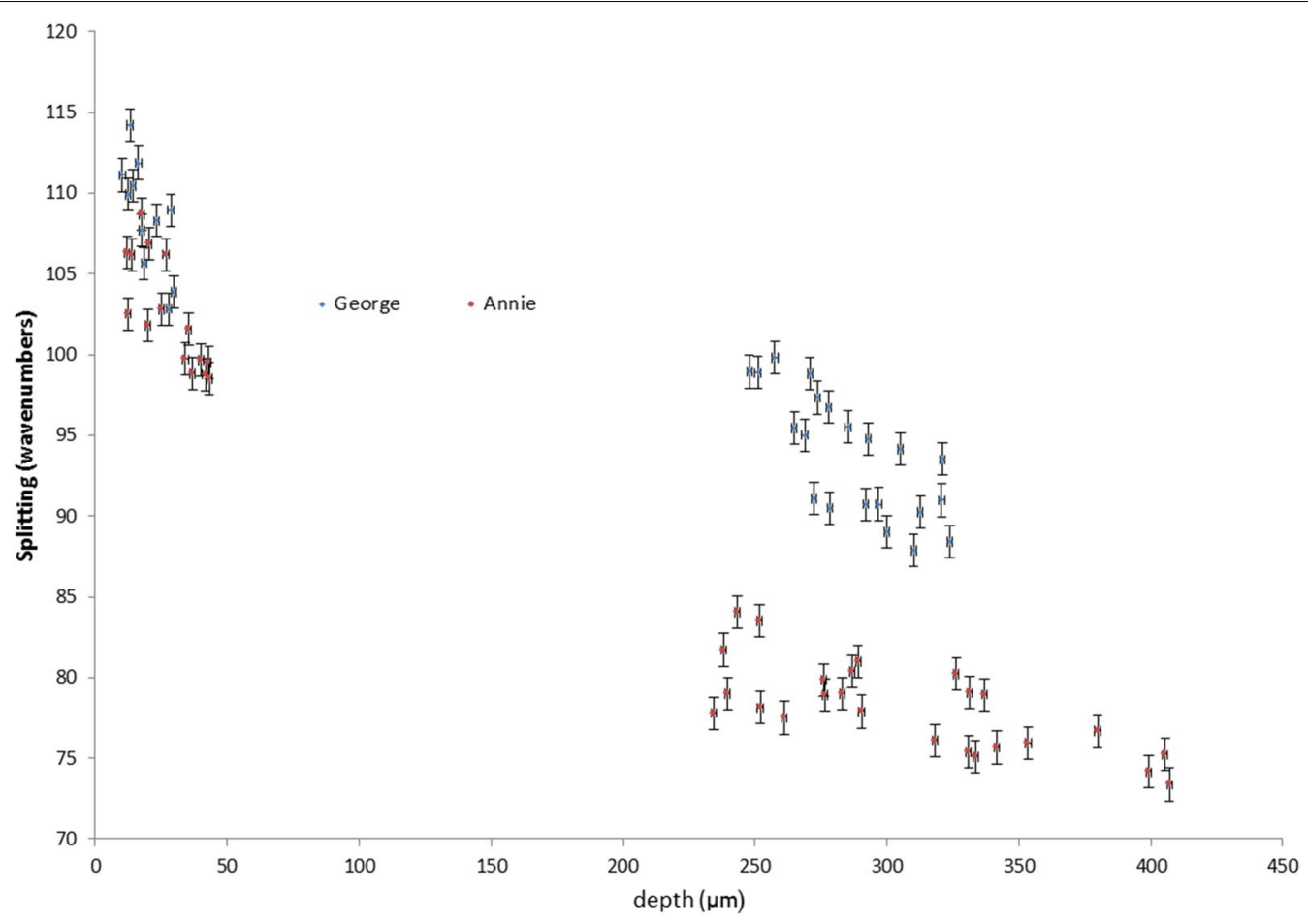

Fig. 3 Degree of degradation (splitting value) versus depth

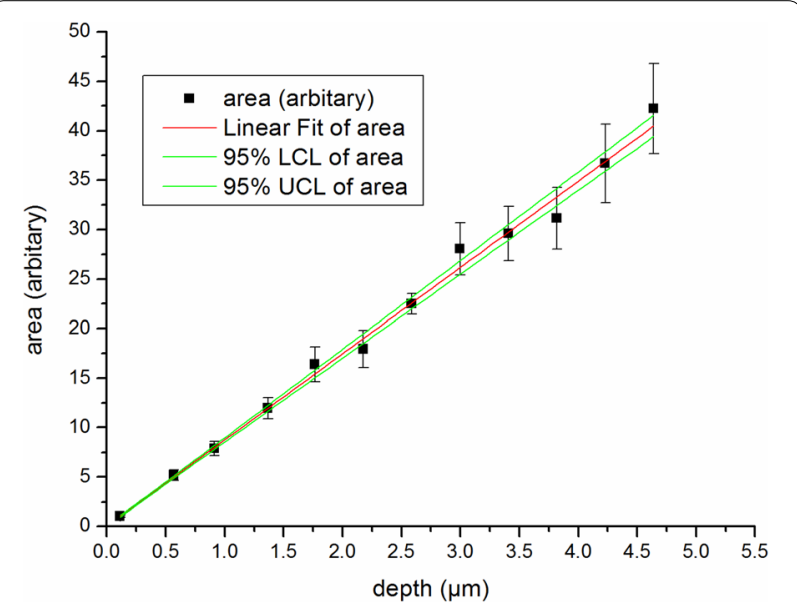

Fig. 4 Depth calibration for Renaissance Wax, UCL-upper confidence limit, LCL-lower confidence limit at 95\% confidence

condensation would be expected to have more impact at the edges or in narrower spaces. Both of these occur more strongly above the matt in Daguerreotype packets.

\section{Renaissance Wax}

The calibration using the $1450 \mathrm{~cm}^{-1}$ absorption in reflection-absorption FTIR on the different depths of Renaissance Wax is shown in Fig. 4.
Each point is the average of eight measurements of each different depth sample. The error bars are 2 standard deviations of the peak area measured. The green lines are the lower and upper confidence limit at $95 \%$ for the calibration generated.

Figure 5 shows representative results of the water depth analysis with subsequent excimer ablations. Those depth segments of the wax with significant water present are marked in blue. The excluded RH values showed similar results and the samples with both the minimum and maximum depth of water present are included in Fig. 5.

There was no statistical difference between the measured depth for water present between any of the 30 analyses taken ( $t$-tests at $99 \%$ confidence interval), indicating that once the $\mathrm{RH}$ exceeds $75 \%$ the phenomena occurs and long exposure to $40 \% \mathrm{RH}$ does not remove any of the water. Based on these results, it is clear that water is present in the lower portion of the wax, adjacent to the steel surface. Full results of the corrosion experiments and their interpretation have been published separately [25]. The localization of the water and particularly evidence that ambient drying does not remove it, was essential in understanding the poor performance of the wax observed. The danger of holding water close to a metal surface has been recognised [26] and whilst poor performance of microcrystalline waxes has been measured [2729 ], the causes have not been investigated previously. 


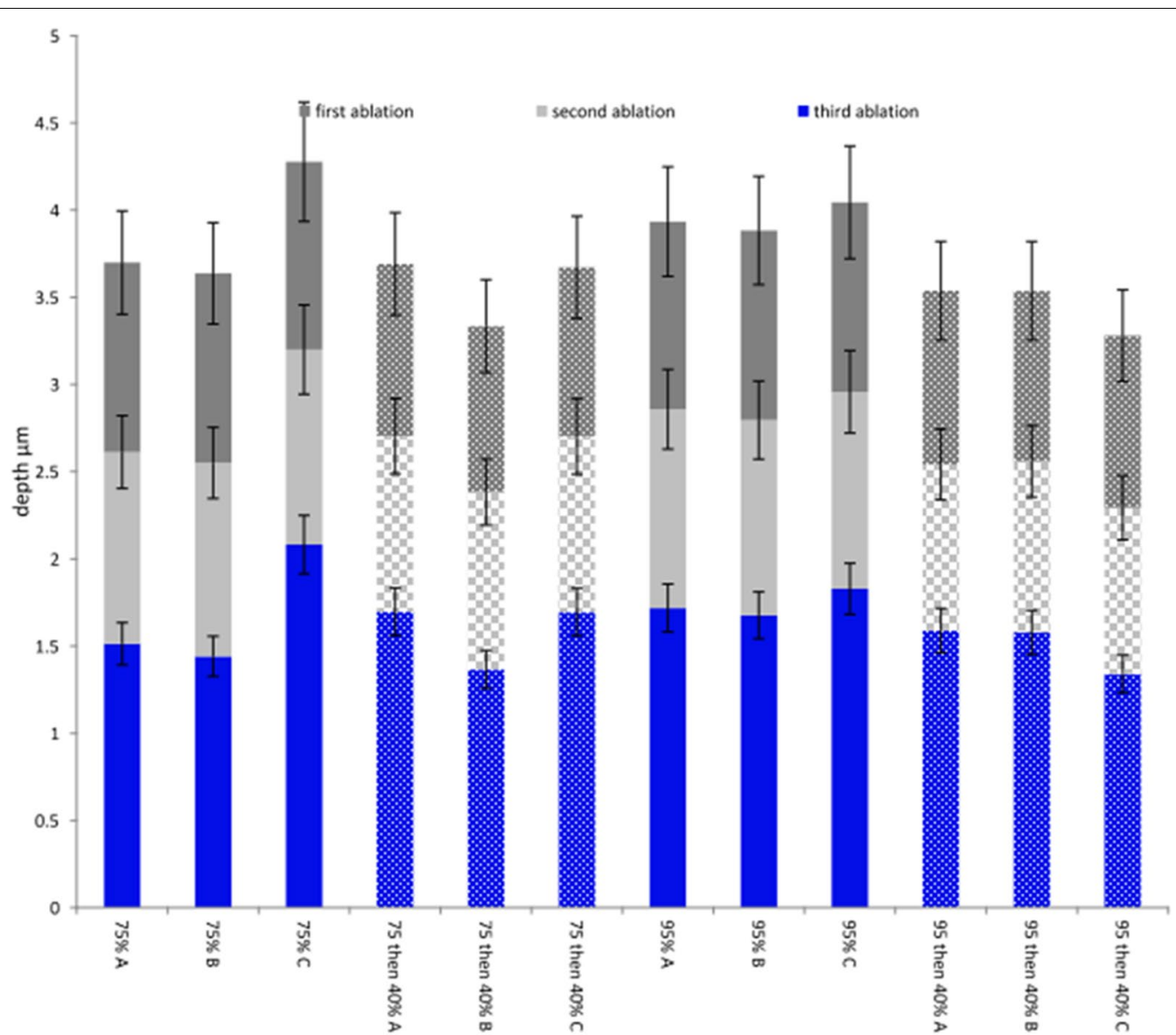

Fig. 5 Ablation sections with (blue) and without (grey) water in Renaissance Wax exposed to different atmospheres

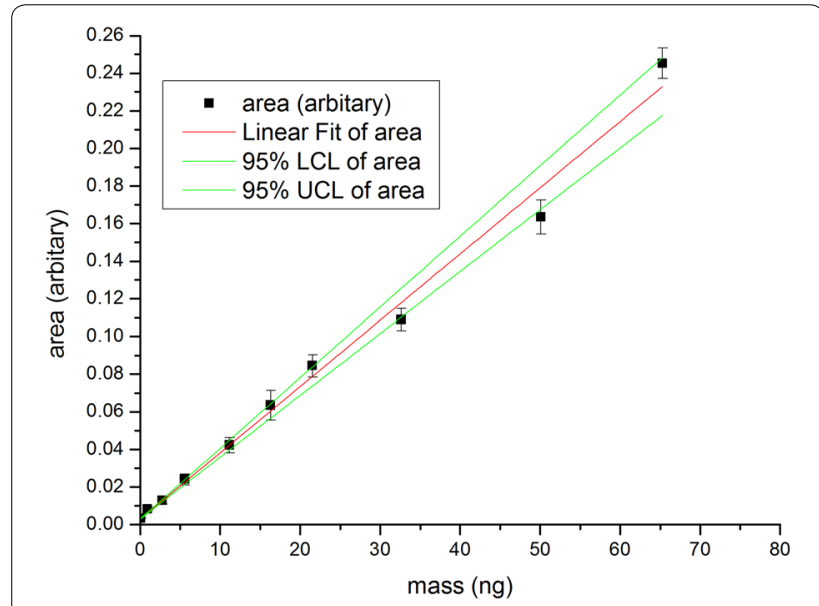

Fig. 6 FTIR calibration for Haze. UCL-upper confidence limit, LCLlower confidence limit at 95\% confidence

\section{Haze}

Results of the PeaSoup haze calibration are shown in Fig. 6.
The detection limit of that $10 \mathrm{MHz}$ crystal and the Open PQC equipment used for measurement is quoted at $1 \mathrm{ng}$. The infra-red produced good spectra at 10 times dilution of this, but not at a 20 times dilution. The detection limit is estimated at $0.1 \mathrm{ng}$, but mass measurement was not possible down to this level. However, the calibration line is a good linear fit at masses from 1 to $65 \mathrm{ng}$. Assuming this is valid, the detection limit is over fifty times lower than the commercial method.

Results of the analyses are shown in Table 1.

Haze deposits were detected in adjacent galleries, which had been invisible to the previous method. This is critical, as the company's literature states 'it leaves behind no residue deposits' [30]. The long term effects of the haze on objects therefore becomes important. The mineral oil has a very low vapour pressure and will not evaporate over several years and probably decades. Repetition of the calibration with the original samples after 12 months, showed no detectable loss of oil. Three obvious mechanisms occur by which the haze residues could have deleterious effects. 
Table 1 Amount of haze detected at different locations

\begin{tabular}{llll}
\hline Slide number & Orientation & Amount of haze (ng) & Observations \\
\hline 1 & Horizontal & $32 \pm 4$ & Very large amount of proteinaceous dust, trace haze \\
2 & Vertical & $24 \pm 3$ & Large amount of proteinaceous dust, some haze \\
3 & Horizontal & $8 \pm 1$ & Very large amount of proteinaceous dust, trace haze \\
4 & Vertical & Below detection & Very large amount of proteinaceous dust \\
5 & Horizontal & $12 \pm 2$ & Very large amount of proteinaceous dust, trace haze \\
6 & Vertical & Below detection & Very large amount of proteinaceous dust \\
7 & Horizontal & $56 \pm 10$ & Small amount of proteinaceous dust, trace haze \\
9 & Vertical & Very weak spectrum & Selow detection
\end{tabular}

- Increased soiling rates: For many surfaces dust particles leave the surface as well as depositing [31], the haze could adhere particles, preventing this happening.

- Increased moisture content: The mineral oil has some hygroscopicity, which may corrode metal surfaces at lower humidities [32] and may increase the degradation rate for many organic materials [33].

- Mould germination at lower RH: for some uncommon mould species this effect has been reported for glycerol [34]. Similar effects may occur with moulds observed on heritage collections.

Potential increased soiling rates were investigated by exposing duplicate glass slides horizontally and vertically (to the floor), in a room and adjacent rooms during haze use for filming (1 day, $300 \mathrm{ml}$ Pea Soup haze) at Eltham Palace. The day after filming, one set of slides was removed and another clean set of slides were exposed adjacent to the first slides. The slides were analysed with automated image analysis under a microscope [35]. Results are shown in Table 2.
As can be seen, there is very low measureable deposition of dust during haze use. The haze droplets are so small $(0.2 \mu \mathrm{m})$, that they would not be expected to be detectable with the system used, which has a minimum particle detection limit of $2 \mu \mathrm{m}$ for spherical particles. For all the slides, the dust deposition rate increased dramatically with the haze residues present. The effect was stronger for vertically oriented slides. This is particularly concerning for fragile surfaces and in historic houses, where housekeeping consumes a very significant part of preventive conservation budgets [36].

Considering increased moisture content for organic materials, the maximum reported deposition rate has been $4 \mu \mathrm{g}$ of haze over a $37.5 \mathrm{~cm}^{2}$ area [21]. Mineral oils generally have very low hygroscopicity, compared to glycerol [37, 38]. Taking the worst case of a glycerol haze, full isotherms (from 10 to $100{ }^{\circ} \mathrm{C}$ ) have been published [38]. Assuming a low density, $60 \mathrm{~g} / \mathrm{m}^{2}$ paper substrate (to give the lowest object mass and hence, proportional glycerol mass), the mass of paper per $37.5 \mathrm{~cm}^{2}$ is $2.25 \mathrm{mg}$. At $50 \%$ $\mathrm{RH}$ a typical paper would have 6 mass percent moisture present, $0.135 \mathrm{mg}$ [39]. The deposited glycerol haze, $4 \mu \mathrm{g}$,

Table 2 Dust deposition during and after haze use

\begin{tabular}{|c|c|c|c|c|}
\hline \multirow[t]{2}{*}{ Location/orientation } & \multicolumn{3}{|l|}{ Dust deposition\% } & \multirow{2}{*}{$\begin{array}{l}\text { Percentage increase } \\
\text { in dust deposition }\end{array}$} \\
\hline & $\begin{array}{l}\text { Directly after filming (1 day } \\
\text { exposure) }\end{array}$ & $\begin{array}{l}\text { After filming } \\
\text { plus } 30 \text { days }\end{array}$ & $\begin{array}{l}\text { New slide ( } 30 \text { days } \\
\text { exposure) }\end{array}$ & \\
\hline Haze room/hor & 0.01 & 2.1 & 1.2 & 175 \\
\hline Haze room/hor & 0.01 & 1.7 & 0.8 & 213 \\
\hline Haze room/ver & 0.00 & 0.09 & 0.03 & 300 \\
\hline Adjacent room A/hor & 0.02 & 2.8 & 1.7 & 165 \\
\hline Adjacent room A/hor & 0.01 & 2.1 & 1 & 210 \\
\hline Adjacent room A/ver & Below detection & 0.11 & 0.03 & 367 \\
\hline Adjacent room B/hor & 0.02 & 1.1 & 0.6 & 183 \\
\hline Adjacent room B/hor & 0.01 & 1.4 & 0.9 & 156 \\
\hline Adjacent room B/ver & Below detection & 0.07 & 0.02 & 350 \\
\hline
\end{tabular}

Hor horizontal, Ver vertical 
would hold $20 \%$ water at equilibrium at $50 \% \mathrm{RH}$, giving an additional moisture load of $0.8 \mu \mathrm{g}$ or $0.59 \%$ of the water already present. This is extremely unlikely to affect physical response or chemical aging.

It is clear haze should be used with caution. Small amounts are deposited in adjacent rooms. The low level deposits increase soiling rates and although a single exposure is unlikely to have adverse effects on organic materials through increased moisture content, multiple exposures will be cumulative. Work is underway investigating the effects on metal corrosion and some literature indicates the potential for mould germination at lower $\mathrm{RH}$ values [34]. Further research is required to fully assess the long term risk.

\section{Conclusions}

More sensitive analysis gives earlier detection of degradation, which is extremely useful for conservation. The more widely used SEM examination of cross sections could not determine a gel layer on the Daguerreotype cover glass surfaces, while using the most surface-sensitive ATR crystal has both determined, and quantified, the gel layer. The enhanced deterioration on the inside glass surfaces indicates the environment is more corrosive inside the Daguerreotype packets than in the showcase. The distribution of deterioration gives important indications on the likely degradation mechanisms. The method is non-invasive and suitable for sound glass. ATR FTIR analysis is limited, in that if the gel layer extends beyond $0.5 \mu \mathrm{m}$, no further changes in FTIR would be seen. The information depth can be extended by using a lower refractive index ATR crystal, such as zinc sulfide, which would extend the information depth to $1.5 \mu \mathrm{m}$, albeit with a reduction in sensitivity. Total reflection FTIR can also be used and has been shown to successfully give decent quality spectra at least on some glasses to a depth of up to $2.04 \mathrm{~mm} \mathrm{[40]} \mathrm{and} \mathrm{is} \mathrm{effective} \mathrm{at} \mathrm{monitoring} \mathrm{the}$ later stages of glass degradation. If sensitive analysis can be repeated at the same point, or using statistical methods across a surface after a time period, real rates of degradation can be analysed. The importance of this cannot be over-emphasised, as surrogate materials are often poor replicas of actual aged historical materials; many heritage environments are complex and their effects are difficult to replicate with simple laboratory experiments.

The assessment of conservation treatment performance is critical to improving conservation practice. The interaction of conservation materials with the environment and artefacts is frequently a surface phenomenon. The identification of liquid waters presence at the steel wax interface contributes to explaining the poor performance of Renaissance Wax and enhanced corrosion of steel coated with it, at higher relative humidities. The effective lack of evaporation, exasperates the situation. Another area where the technique can contribute is identifying unknown lacquers and conservation materials. Conservation records are rare before the 1970s and as several materials have been in very long term use, their performance after many decades of natural aging is of major interest. Identification is the first stage in this process in the absence of treatment records and reflectance FTIR is uniquely suited to this task for coatings on metals.

For monitoring, sensitivity can be critical. In many instances 'safe'and 'damaging' concentrations have not been defined, and using the detection limit of a particular technique can be misleading. In the case presented, the 50 times increase in detection limit identified haze residues. These residues adjacent to objects in galleries away from the haze application means the mitigation applied was not fully successful and has potential long term impacts on collections.

\section{Abbreviations \\ ATR: attenuated total reflection; FTIR: Fourier transform infra-red; IR: infra-red; SEM: scanning electron microscopy; SEM-EDX: scanning electron micros- copy-energy dispersive $\mathrm{x}$-ray analysis; UV: ultra-violet.}

\section{Acknowledgements}

We acknowledge Marianne Odlyha for kind access to the Amplif-IR equipment, and UCL for use of the Excimer laser.

\section{Authors' contributions}

DT undertook all analysis and was principle researcher for the glass and wax projects. BP initiated the haze monitoring, deployed the glass slides and discussed the results and their interpretation. He provided access to the FTIR microscope used in the glass project. Both are IRUG directory board members. Both authors read and approved the final manuscript.

\section{Funding}

All funding was from internal English Heritage and Victoria and Albert Museum Research funds.

\section{Availability of data and materials}

The datasets used and/or analysed during the current study are available from the corresponding author on reasonable request. All the materials analysed for the study are being curated by English Heritage.

\section{Competing interests}

The authors declare that they have no competing interests.

\section{Author details}

${ }^{1}$ English Heritage, Rangers House, London SE108QX, UK. ${ }^{2}$ Victoria and Albert Museum, Cromwell St, London SW7 2RL, UK.

Received: 5 Auqust 2019 Accepted: 11 January 2020

Published online: 23 January 2020

\section{References}

1. Ryan JL, MCPhail DS, Rogers PS, Oakley VL. In Preprints of ICOM CC 11th Triennial Meeting: Edinburgh, Lyon 1996: Preprints. London: James and James; 1996. p. 836-44.

2. Hallett K, Thickett D, McPhail D, Chater R. Application of SIMS to Silver Tarnish at the British Museum. Appl Surf Sci. 2003;203-204:789-92. 
3. Pouliot B, Matsen C, Mass J, Donnelly W, Andrews K, Beard M. Three decades later: a status report on the silver lacquering program at Winterthur. In: Objects specialty group postprints, vol. 20. 2013. p. 32-48.

4. Derrick MR, Stulik D, Landry JM. Infrared spectroscopy in conservation science. Los Angeles: The Getty Conservation Institute; 1999.

5. Margariti C. The application of FTIR microspectroscopy in a non-invasive and non-destructive way to the study and conservation of mineralised excavated textiles. Herit Sci. 2019;7:63. https://doi.org/10.1186/s4049 4-019-0304-8

6. Yan Y, Wen C, Jin M, et al. FTIR spectroscopy in cultural heritage studies: non-destructive analysis of Chinese Handmade Papers. Chem Res Chin Univ. 2019;35:586-91. https://doi.org/10.1007/s40242-019-9026.

7. Thickett D. Basic chemical mechanisms indoors. In: Havermanns J, Adrians A, editors. Basic environmental mechanisms affecting cultural heritage. Florence: Nardini Editore; 2010. p. 69-74.

8. Shashoua Y, Berthelsen MB, Lund Degn N, Ole F. Raman and ATR-FTIR spectroscopies applied to the conservation of archaeological Baltic amber. J Raman Spectrosc. 2007;37:1221-7.

9. Picollo M, Bartolozzi G, Cucci C, Galeotti M, Marchiafava V, Pizzoc B. Comparative study of fourier transform infrared spectroscopy in transmission, attenuated total reflection, and total reflection modes for the analysis of plastics in the cultural heritage field. Appl Spectrosc. 2014;68(4):389-97.

10. Thickett D, Pretzel B. Micro-spectroscopy: a powerful tool to understand deterioration. E-Preserv Sci. 2010;7:158-64.

11. Tennent NH. Weeping glass: the identification of ionic species on the surface of vessel glass using ion chromatography. In: Recent advances in glass and ceramics conservation. Amsterdam 2016: Preprints. Paris: ICOM; 2016. p. 123-33

12. Lamain BvB MR, Verhaar G, Tennent NH. The development of an ion chromatography protocol for detecting the early stages of glass degradation. In: Recent advances in glass, stained-glass, and ceramics conservation. Amsterdam 2013: Postprints. Amsterdam: Stichting Promotie Archeologie 2013. p. 303-11.

13. Harrick NJ, du Pre FK. Effective thickness of bulk materials and of thin films for internal reflection spectroscopy. Appl Opt. 1966:5:1739-43.

14. MacDonald SA, Schardt CR, Maisiello DJ, Simmons JH. Dispersion analysis of FTIR reflection measurements in silicate glasses. J Non-Cryst Solids. 2000;275:72-82

15. Harris R. Metalwork. The national trust manual of housekeeping. Amsterdam: Butterworth Heinemann; 2006. p. 258-9.

16. Thickett D, Stanley B. Corrosion protection for complex large equipment. In: Big Stuff. Lewarde, (France) 2015. https://bigstuff.omeka.net/items/ show/1bigstuff2016. Accessed 10 July 2019.

17. Horie V. Materials for conservation: organic consolidants, adhesives and coatings. London: Elsevier; 2013.

18. Teule $\mathrm{R}$, Scholten $\mathrm{H}$, van den Brink OF, Heeren RMA, Albuquerque MB. Controlled UV laser cleaning of painted artworks: a systematic effect study on egg tempera paint samples. J Cult Herit. 2003:4(Sup 1):209-15

19. Thickett D. Analysis of iron corrosion products Fourier Transform Infra-red and Raman spectroscopies. In: Postprints of IRUG 6, Padua 2003: Postprints. Padua: II Prato; 2005. p. 86-93.

20. Thickett D. Post excavation changes and preventive conservation of archaeological iron. PhD dissertation, School of Biological and Chemical Sciences, Birkbeck College, University of London, 2012.

21. Blades N. Test of Pea Soup Phantom Pro hazer at the Orangery, Ham. National Trust Internal Report 2010. Personal communication. 2018.

22. Frankel GS, Vienna JD, Lian J, Scully JR, Gin S, Ryan JV, Wang J, Kim SH, Wind I W, Du J. A comparative review of the aqueous corrosion of glasses, crystalline ceramics, and metals. Npj Mater Degrad. 2018;2:15. https://doi. org/10.1038/s41529-018-0037-2.

23. Kunicki-Goldfinger JJ. Unstable historic glass: symptoms, causes, mechanisms and conservation. Stud Conserv. 2008;53(Supplement-2):47-60. https://doi.org/10.1179/sic.2008.53.supplement-2.47.

24. Barger MS, Smith DK, White WB. Characterization of corrosion products on old protective glass, especially daguerreotype cover glasses. J Mater Sci. 1989;24:1343-56.

25. Thickett D, Stanley B. The use and mis-use of renaissance wax. In: Metal 2019. Neuchatel, 2019: Preprints. Neuchatel:ICOM-CC and HE-Arc CR 2019.

26. Watkinson D. Preservation of metallic cultural heritage. In: Cottis RA, editor. Shreir's corrosion. 4th ed. London: Elsevier; 2010. p. 3307-40.

27. Argyropoulous V. Metals and museums in the mediterranean; protecting, preserving and interpreting. Athens: TEl of Athens; 2008.

28. Otieno-Alego V, Hallam D, Viduka A, Heath G, Creagh D. Electrochemical impedance studies of the corrosion resistance of wax coatings on artificially patinated bronze. In: Metal 98 . Proceedings of the international conference on metal conservation, Draguignan-Figanières (France). 1998: Preprints. London: James \& James 1998. p. 309-14.

29. Hallam D, Thurrowgood D, Otieno-Alego V, Creagh DAV. Studies of commercial protective petrochemical coatings on ferrous surfaces of historical and museum objects. In: Metal 2001: proceedings of the international conference on metals conservation, Santiago (Chile) 2001: Preprints. Welshpool: Western Australian Museum 2004. p. 297-303.

30. Slvision https://sIvision.co.uk/pea-soup-phantom-hazer-hire. Accessed 12 July 2019.

31. Kibrya R, Adams S, Brimblecombe P. A particle accumulation study during the reconstruction of The Great Court, British Museum. J Cult Herit 2002;3(4):283-7. https://doi.org/10.1016/S1296-274(02)01237-2.

32. Leygraf C, Odnevall Wallinder I, Tidblad J, Graedel T. Atmospheric corrosion. 2nd ed. London: Wiley; 2016.

33. Michalski S. Double the life for each five-degree drop, more than double the life for each halving of relative humidity. In: ICOM Committee for Conservation, 13th Triennial Meeting, Rio de Janeiro 2002: Preprints. London: James and James; 2002. p. 66-71.

34. Hallsworth JE, Magan N. Manipulation of intracellular glycerol and erythritol enhances germination of conidia at low water availability. Microbiology. 1995;141:1109-15.

35. Howell D, Brimblecombe P, Lloyd H, Frame K, Knight B. Monitoring dust in historic houses. Conservation science 2002: preprints. London: Archetype 2002. p. 8-10.

36. Lloyd H, Brimblecombe P, Lithgow K. Economics of dust. Stud Conserv. 2007:52:135-45.

37. Echin Al, Novosartov GT, Popova EA. Hygroscopicity of synthetic oils. Chem Technol Fuels Oils. 1981;17(4):198-200.

38. Miner CS, Dalton NN. Glycerol. New York: Reinhold; 1953.

39. Paltakari JT, Karlsson MA. Determination of specific heat for dry fibre material. In: 82nd Annual meeting, Technical Section, CPPA 30 Jan-2 Feb Montreal Canada 1996: Postprints Montreal: CPPA; 1996. p. B117-B120.

40. Thickett D, Cheung CS, Liang H, Twydle J, Gr Maev R, Gavrilov D. Using non-invasive non-destructive techniques to monitor cultural heritage objects. Insight. 2017:59(1):1-5.

\section{Publisher's Note}

Springer Nature remains neutral with regard to jurisdictional claims in published maps and institutional affiliations. 